Results One dog in $\mathrm{R}$ group died for anaesthesia, another dog in $\mathrm{Re}$ group had pacemaker problem that required us to remove them from the study. The SCL shortened after pacing $(510 \pm 24 \mathrm{~ms} v \mathrm{~s} 430 \pm 18 \mathrm{~ms}$, $380 \pm 26 \mathrm{~ms}, \mathrm{p}<0.05)$, ERP shortened $(146 \pm 18 \mathrm{~ms}$ vs $125 \pm 18 \mathrm{~ms}$, $115 \pm 19 \mathrm{~ms}, \mathrm{p}<0.01)$, dERP increased. The expression of $\mathrm{Cx} 40$ increased significantly in both experiment groups (1017.23 \pm 314.46 Int $\times \mathrm{mm}^{2}$ vs $1709.43 \pm 429.88 \mathrm{Int} \times \mathrm{mm}^{2}, 2956.05 \pm 829.38 \mathrm{Int} \times \mathrm{mm}^{2}$ $\mathrm{p}<0.01)$, the expression of $\mathrm{Cx} 43$ increased significantly in above groups (915.21 $\pm 338.93 \mathrm{Int} \times \mathrm{mm}^{2}$ vs 1859.94 \pm 412.11 Int $\times \mathrm{mm}^{2}$, 3048.83 \pm 931.35 Int $\left.\times \mathrm{mm}^{2}, \mathrm{p}<0.01\right)$. The length and width of GJ in pulmonary vein sleeves shortened (L: $0.381 \pm 0.034 \mu \mathrm{m}$ vs $0.390 \pm 0.117 \mu \mathrm{m}, \quad 0.260 \pm 0.069 \mu \mathrm{m} ; \quad$ W: $\quad 23.29 \pm 3.70 \mathrm{~nm} \quad$ vs $22.38 \pm 2.46 \mathrm{~nm}, 18.54 \pm 2.56 \mathrm{~nm}, \mathrm{p}<0.05)$, and the Termination/Side $(\mathrm{T} / \mathrm{S})$ ratio of $\mathrm{Cx} 40$ and $\mathrm{Cx} 43$ decreased $(\mathrm{Cx} 40: 1.34 \pm 0.11$ vs $1.16 \pm 0.07$, $0.98 \pm 0.06$; $\mathrm{Cx} 43$ : $1.27 \pm 0.09$ vs $1.10 \pm 0.07,0.90 \pm 0.09, \mathrm{p}<0.05)$.

Conclusions The vagus nerve originated from SVC-Ao fat pad plays a role in pulmonary vein reconstitution, and these are part of basal elements for the development and maintenance of experimental atrial fibrillation.

\section{e0019 STUDY ON THE CHANGES OF MATRIX METALLOPROTEINASE-9 AND HIGH SENSITIVE C-REACTIVE PROTEIN IN PATIENTS WITH CAROTID INTIMA-MEDIA THICKNESS}

doi:10.1136/hrt.2010.208967.19

Yun-Kai Wang, Yun-Fen Wei, Meng-Hong Wang, Ze-Oi Zheng, Lin-Li Wang, Yin- Sheng Wu. Department of Cardiology, the First Affiliated Hospital of Nan Chang University

Objective To investigate the relationship between matrix metalloproteinase- 9 , high sensitive C-reactive protein and carotid intimamedia thickness.

Methods 180 patients were selected. High-resolution ultrasound was used to scan carotid, brachial arteries of all patients in order to check and measure carotid intima-media thickness (CIMT), plaques of carotid arteries and diameter of brachial arteries at rest. Patients were divided into four groups: the normal carotid intima group, the difficuse proliferative carotid intima group, the stable plaque group and the unstable plaque group. Serum levels of MMP-9, hsCRP were determined with immunological method. The relationship between the results measured by ultrasound instrument and the concentrations of MMP-9, and hsCRP was analysed.

Results The serum levels of MMP-9 and hsCRP in the proliferative carotid intima group were higher than in the normal carotid intima group $(p<0.01)$. The serum levels of MMP-9 and hsCRP in the unstable plaque group and in the stable plaque group were higher than in difficuse proliferative carotid intima group $(p<0.05)$. The serum levels MMP-9 and hsCRP in the unstable plaque group were higher than in the stable plaque group $(p<0.05)$.

Conclusion The increased concentrations of MMP-9 and hsCRP were closely correlated with the increment of carotid intima-media thickness, the unsteady of plaque.

\section{e0020 COMPOUND HETEROZYGOUS NOVEL SPLICING MUTATION D202SP AND MISSENCE MUTATION G272D ON KCN01 CAUSED JERVEL AND LANGE-NIELSEN SYNDROME IN A CHINESE FAMILY}

doi:10.1136/hrt.2010.208967.20

Li Cuilan, Liu Wenling, Liang Ruijuan, Qiu Xiaoliang, Liu Rui, Li Lei, Li Jianfeng, Wang Jun, Hu Dayi. Department of Thoracic Surgery, Peking University People's Hospital, Beijing, P.R. China

Background Long OT syndrome (LOTS) is an inherited cardiac disorder characterised by OT interval prolongation on ECG, ventricular arrhythmias and sudden death. Two forms have been identified: autosomal dominant Romamo-Ward syndrome (RWS) without deafness and autosomal recessive Jervell and Lange-Nielsen syndrome (JLNS) with deafness.

Methods A Chinese family with JLNS was identified. Family members were diagnosed based on the presence of a OT interval prolongation on 12-lead ECG and a history of syncope, palpatation and deafness. Mutational screening in the KCNQ1 potassium channel gene was performed by direct DNA sequence analysis and Blast with Results: A female 12-y-o proband and her 6-y-o brother were diagnosed with JLNS in May of 2009. The OTc were $0.59 \mathrm{~s}$ and $0.60 \mathrm{~s}$ for the girl and boy, respectively. Both patients had their first syncope at the age of 2. The QTc for parents was normal. The mutation detection showed two mutations: one is a novel splicing mutation, a $A$ to $G$ change in the position of two bases before exon 3 (A 605-2 $A \rightarrow G$ ), in the acceptor site of intron 2, which is always A followed by G. Another one is a $\mathrm{G}$ to A change at position of 815 , which is a known missense mutation G272D reported before in a RWS European patient. Both JLNS patients were compound heterozygous for these two mutations D202sp and G272D. The father carries a heterozygous D202sp only, while the mother carries a heterozygous G272D. These two mutation were absent in 100 control alleles. The parents' marriage was not consanguineous. Because of the early age of first syncope and the less effectiveness of b-blocker for JLNS, videoassisted thoracoscopic left cardiac sympathetic denervation (LCSD) was performed successfully in June of 2009. Both patients were syncope free during 1-year follow-up after surgery at the dosage of 2.2 and $1.6 \mathrm{mg} / \mathrm{Kg}$ of proparanolol for the girl and boy.

Conclusion Our results suggest that the compound heterozygous mutation D202sp and G272D caused JLNS in the siblings of this Chinese family. To our best knowledge, this is the first report of compound heterozygous splicing and missense mutation to induce JLNS so far. The results expand the spectrum of KCNQ1 mutations causing RWS and JLNS. Further mechanism exploration will deep our understanding of this rare disease.

\section{e0021 A NOVEL APPROACH OF PROTEOMICS TO STUDY THE MECHANISM OF ACTION OF GRAPE SEED PROANTHOCYANIDIN EXTRACTS ON AORTIC ARTERIOSCLEROSIS IN DIABETIC RATS}

doi:10.1136/hrt.2010.208967.21

Li Bao-Ying, Li Xiao-Li, Gao Hai-Qing, Cheng Mei, Xu Ling, Li Xian-Hua. Qi-Lu Hospital of Shandong University

Objective Diabetic macrovascular complications are the leading cause of mortality in diabetic patients. To prevent the development of this disease and to improve advanced arteriosclerosis, effective therapies directed towards the key molecular target are required. Grape seed proanthocyanidin extracts (GSPE) have been reported to be effective in treating arteriosclerosis, while little is known about the functional protein changes.

Methods We used streptozotocin to induce diabetic rats. GSPE ( $250 \mathrm{mg} / \mathrm{kg}$ body weight/day) were administrated to diabetic rats for 24 weeks. Serum glycated haemoglobin and advanced glycation end products (AGEs) were determined. Electronic microscope was used to observe the changes of aortic ultrastructure. Immunohistochemistry was used to evaluate the receptor of advanced glycation end products (RAGE) protein expression in aortic tissue. Consequently, 2-D difference gel electrophoresis and AutoFlex martix-assisted laser desorption/ ionization time-of-flight mass spectrometry with LIFT technology or liquid chromatography electrospray ionisation mass spectrometry/ mass spectrometry were used to investigate aortic protein profiles among the control, untreated, and GSPE treated diabetic rats.

Results GSPE significantly decreased aortic PWV, blood pressures, aortic medial thickness $(p<0.05)$, and inhibited the migration of 